\title{
Publisher Correction: Ensemble learning of diffractive optical networks
}

Md Sadman Sakib Rahman, Jingxi Li $\mathbb{1}$, Deniz Mengu, Yair Rivenson and Aydogan Ozcan (D)

\section{Correction to: Light: Science \& Applications \\ https://doi.org/10.1038/s41377-020-00446-w \\ published online 11 Jan 2021}

After publication of this article ${ }^{1}$, it is noticed that the article contained some typographical errors:

1. In Table 1 caption text, "D2NN" should have been " $\mathrm{D}^{2} \mathrm{NN}$," where 2 is superscript.

2. In the "Materials and Methods" section, "where and $\beta$ are..." should read as "where $\alpha$ and $\beta$ are...".
These typographical errors were introduced during the editorial production and were not part of the authors' original manuscript.

Published online: 07 February 2021

\section{Reference}

1. Rahman, M. S. S., Li, J. \& Mengu, D. et al. Ensemble learning of diffractive optical networks. Light Sci. Appl. 10, 14, https://doi.org/10.1038/s41377-020-00446-w (2021). 